'The updated examination

embraces a carefully defined

clinically-based curriculum.

\title{
MFDS (2007): \\ 'a structured and beneficial measure of achievement, underpinning progression in clinical practice'
}

\author{
Jonathan Cowpe outlines the recent changes that have occurred to the MFDS qualification \\ from the Royal College of Surgeons of Edinburgh and the Royal College of Physicians and \\ Surgeons of Glasgow.
}

\section{MFDS}

The existing MFDS/MFD (2001), managed jointly by the Royal College of Surgeons of Edinburgh (RCS Ed) and the Royal College of Physicians and Surgeons of Glasgow (RCPSG), has undergone a redevelopment and refinement over the last two years. The objective has been to keep pace with the new standards of assessment, to ensure it remains educationally sound and fit for purpose.

The updated examination embraces a carefully defined clinically-based curriculum. It provides dentists with a valid and reliable assessment of the knowledge, understanding and competence that should underpin their clinical practice and enable them to deliver the best possible service for their patients. Usually taken over a period of two years following graduation, it should demonstrate that their clinical expertise has reached an appropriate level to enable them to move into further training in their chosen field.

\section{BENEFIT TO THE TRAINEE}

The vast majority of dental graduates currently undertake a period of training equivalent to two years of general professional training (GPT). As 'Modernising dental careers' unfolds, these will become foundation training years, similar to the two-year foundation training programme undertaken in medicine and surgery prior to selection into specialty training.

In December 2005, the GDC ${ }^{1}$ stated that candidates will be eligible for consideration for entry into a specialist training programme if they:

- Are registered with the GDC and fit to practise

- Can demonstrate that they have the required broad-based training, experience and knowledge to enter the training programme.

Success in the MFDS will demonstrate the acquisition of a broad-based knowledge and understanding. Together with other markers of progress through GPT, including work-based assessments, it should provide candidates with a portfolio of evidence demonstrating their suitability to enter specialist training. It will be a measure of the clinical achievement of trainees beyond the primary qualification, and in so doing confirm their commitment to continuing professional development.

\section{FORMAT OF THE EXAMINATION}

The examination's learning objectives aim to embrace the UK Curriculum for Foundation Training, ${ }^{2}$ and the exam itself is in two parts.

\section{Part 1 - written}

Part 1 aims to test the range of knowledge and understanding that underpins direct patient care. A mixture of single best answer, extended matching and multiple short answer questions are utilised. The attributes of problem solving, evaluation of evidence, critical reading, up-to-date knowledge and management of the whole patient will be assessed 
MFDS (2007) provides dental

trainees with a modern, credible

and worthwhile measure of their

progression following undergraduate

training.' using a multiple short answer assessment format.

\section{Part 2 - objective structured clinical} examination (OSCE)

Part 2 aims to test the trainee's clinical competence by assessing the knowledge and understanding required to manage a range of common conditions and in addition, the trainees' communication skills. The OSCE 'stations' will simulate the types of patient consultation that confront candidates during their training and will permit assessment of the candidate's:

- Ability, through appropriate questioning of a patient, to elicit information which will lead to a diagnosis

- Ability to behave in a professional and caring manner; be receptive to the patient's needs; be conscious of his/her expectations; and have respect for confidentiality and autonomy. It will assess how the candidate interacts with his/her patients

- Ability, by explaining the diagnosis and treatment plan, to convey information to patients; to discuss treatment options in partnership with the patient; and to advise, educate and ensure that the patient understands

- Knowledge of a range of clinical management issues through a range of scenarios which they are likely to encounter in their daily practice during the foundation years.

The Postgraduate Medical Education and Training Board (PMETB) oversees medical postgraduate assessment. Its principles and guidance address issues that are also relevant to dentistry. To that end, the RCS Ed and RCPSG are ensuring that their examinations meet the robust and structured quality assurance benchmarks identified and stated through PMETB documentation.

\section{ELIGIBILITY TO TAKE THE EXAMINATION}

- Part 1 may be taken at any time after gaining a primary dental qualification

- Part 2 may be taken after gaining a pass in Part 1 and having undertaken 12 months' postgraduate dental experience in clinical dentistry.

IN SUMMARY

- MFDS (2007) provides dental trainees with a modern, credible and worthwhile measure of their progression following undergraduate training. It is an assessment which embraces a carefully defined clinically-based curriculum. Working through the curriculum should stimulate trainees to achieve their potential by extending their knowledge of applied clinical science, taking their clinical experience, skills and understanding to a higher level, and, in so doing, providing a springboard for the next stage of their careers

- Please consult the Regulations and Guidance to Candidates on the College websites, where a series of 'frequently asked questions' are also available - www.rcsed.ac.uk or www.rcpsg.ac.uk.

\section{J. G. Cowpe}

Dean, Faculty of Dental Surgery of the Royal College of Surgeons of Edinburgh

1. General Dental Council. Specialist lists review group final report. London: GDC, 2005.

2. Department of Health (England). A curriculum for UK dental foundation programme training. London: Department of Health, 2006.

DOI: $10.1038 /$ bdj.2007.910 